\title{
Acronyms, abbreviations and common notation
}

\section{Scientific abbreviations}

\begin{tabular}{|c|c|}
\hline $\operatorname{AAM}(\mathrm{F})$ & Atmospheric Angular Momentum (Function) \\
\hline AMF & Angular Momentum Function \\
\hline $\mathrm{CIO}$ & $\begin{array}{l}\text { Two meanings: Celestial Intermediate Origin / Conventional Inter- } \\
\text { national Origin }\end{array}$ \\
\hline CIP & Celestial Intermediate Pole \\
\hline CRF & Celestial Reference Frame \\
\hline CRS & Celestial Reference System \\
\hline GCRS & Geocentric Celestial Reference System \\
\hline cpy & cycle per year \\
\hline cpd & cycle per day \\
\hline DORIS & Doppler Orbitography by Radiopositioning Integrated on Satellite \\
\hline EAMF & Equatorial Angular Momentum Function \\
\hline EEAMF & Effective Equatorial Angular Momentum Function \\
\hline ECCO & Estimating the Circulation and Climate of the Oceans \\
\hline EOP & Earth Orientation Parameters \\
\hline FCN & Free Core Nutation \\
\hline FWHM & Full Width at Half Maximum \\
\hline GCM & Global Circulation Model \\
\hline GLONASS & GLObalniy NAvigationniy Sputnikoviy System \\
\hline GNSS & Global Navigation Satellite System \\
\hline GPS & Global Positioning System \\
\hline $\operatorname{HAM}(\mathrm{F})$ & Hydrological Angular Momentum (Function) \\
\hline IB & Inverted Barometer (response of the oceans) \\
\hline ICRF & International Celestial Reference Frame \\
\hline ITRF & International Terrestrial Reference Frame \\
\hline IVS & International VLBI Service \\
\hline LOD & Length Of Day (offset of the) \\
\hline mas & milliarcsecond \\
\hline NIB & Non Inverted Barometer (response of the oceans) \\
\hline $\mathrm{OAM}(\mathrm{F})$ & Oceanic Angular Momentum (Function) \\
\hline SLR & Satellite Laser Ranging \\
\hline TAI & Temps Atomique International \\
\hline TIO & Terrestrial Intermediate Origin \\
\hline TPW & True Polar Wander \\
\hline TRF & Terrestrial Reference Frame \\
\hline TRS & Terrestrial Reference System \\
\hline ATRS & Auxiliary Terrestrial Reference System \\
\hline UTC & Universal Time Coordinated \\
\hline
\end{tabular}


UT1

Universal Time 1 (Earth rotation time scale)

VLBI

Very Long Baseline Interferometry

\section{Organisations}

CNES

ECMWF

GFZ

IDS

IERS

IGN

IGS

ILRS

MIT

NCEP
Centre National d'Etudes Spatiales

European Centre for Meteorological Weather Forecasts

GeoForschungZentrum

International DORIS Service

International Earth Rotation and reference system Service Institut National de l'Information Géographique et Forestière International GNSS Service

International Laser Ranging Service

Massachusetts Institute of Technology

National Centre for Environmental Prospect

\section{Notations}

$A$
$B$
$C$
$\bar{A}=(A+B) / 2$
$e=(C-\bar{A}) / \bar{A}$
$\lambda_{A}$
$A_{m}$
$C_{m}$
$e_{m}=\frac{C_{m}-A_{m}}{A_{m}}$
$A_{f}$
$C_{f}$
$e_{f}=\frac{C_{f}-A_{f}}{A_{f}}$
$R_{f}$
$G$
$G M_{\oplus}$
$M_{\oplus}$
$J_{2}=\frac{C-\bar{A}}{M_{\oplus} R_{e}^{2}}$
$g$
$R_{e}$
$r_{0}$
$f$
$\Omega$

Small equatorial principal moment of inertia Large equatorial principal moment of inertia Axial principal moment of inertia

Mean equatorial principal moment of inertia Dynamical ellipticity Longitude of the principal axis of inertia A Equatorial principal inertia moment of the mantle Axial principal inertia moment of the mantle Dynamical ellipticity of the mantle Equatorial principal inertia moment of the fluid core Axial principal inertia moment of the fluid core Dynamical ellipticity of the core Core equatorial radius Gravitational constant Geocentric constant of gravitation Earth's mass Coefficient of the geopotential degree 2 spherical harmonic Gravity field at Earth's surface Earth's mean equatorial radius Earth's mean radius Geometrical flattening Mean Earth's angular velocity 


$\begin{array}{ll}m_{1}, m_{2} & \begin{array}{l}\text { Equatorial cosine direction of the Earth's instantaneous rotation } \\ \text { vector in the TRS }\end{array} \\ m_{1}+m_{1}^{f}, m_{2}+m_{2}^{f} & \begin{array}{l}\text { Equatorial cosine direction of the inner core rotation vector in the } \\ \text { TRS }\end{array} \\ m=m_{1}+i m_{2} & \text { Complex rotation pole coordinate } \\ p=x-i y & \text { Complex coordinate of the CIP } \\ T_{c} & \text { Chandler wobble's period } \\ f_{c} & \text { Chandler wobble's frequency } \\ \sigma_{c}\left(\tilde{\sigma}_{c}\right) & \text { Chandler wobble's angular frequency (complex) } \\ \sigma_{f}\left(\tilde{\sigma}_{f}\right) & \text { Free core nutation angular frequency (complex) in the TRS } \\ Q & \text { Quality factor of the Chandler wobble } \\ k_{2} & \text { Solid Earth Love number } \\ k_{o} & \text { Oceanic Love number } \\ k_{2}^{\prime} & \text { Loading Love number for the mantle } \\ k_{f}^{\prime} & \text { Loading Love number for the core } \\ k_{s}=\frac{3 G(C-\bar{A})}{\Omega^{2} R_{e}^{5}} & \text { Secular Love number } \\ h_{2} & \text { Displacement Love number for the solid Earth } \\ \rho_{o} & \text { Ocean density } \\ \rho_{\oplus} & \text { Earth's density } \\ c=c_{13}+i c_{23} & \text { Off-diagonal inertia moment } \\ h=h_{1}+i h_{2} & \text { Equatorial relative angular momentum } \\ \chi_{G} & \text { Geodetic excitation } \\ \chi_{A / O / H} & \text { Angular momentum function of the atmosphere/oceans/hydro- } \\ \chi_{A / O / H}^{e} & \text { sphere } \\ & \text { Effective angular momentum function of the atmosphere/oceans/ } \\ & \text { hydrosphere }\end{array}$


